\title{
Epidemiology of non-alcoholic fatty liver disease- related hepatocellular carcinoma: a western perspective
}

\author{
Ann Farrell, ${ }^{1,2}$, Marno Ryan ${ }^{1,2}$, Jessica Howell ${ }^{1,2,3}$ \\ 'Department of Gastroenterology, St. Vincent's Hospital Melbourne, Melbourne 3065, Australia. \\ 2Department of Medicine, The University of Melbourne, Melbourne 3010, Australia. \\ ${ }^{3}$ Disease elimination program, Burnet Institute, Melbourne 3004, Australia.
}

Correspondence to: Dr. Ann Farrell, Department of Gastroenterology, St Vincent's Hospital Melbourne, Fitzroy 3065, Australia. E-mail: ann.farrell@svha.org.au

\begin{abstract}
How to cite this article: Farrell A, Ryan M, Howell J. Epidemiology of non-alcoholic fatty liver disease-related hepatocellular carcinoma: a western perspective. Hepatoma Res 2020;6:18. http://dx.doi.org/10.20517/2394-5079.2019.019
\end{abstract}

Received: 14 Oct 2019 First Decision: 3 Feb 2020 Revised: 27 Feb 2020 Accepted: 9 Mar 2020 Published: 23 Apr 2020

Science Editor: Darrell Crawford Copy Editor: Jing-Wen Zhang Production Editor: Jing Yu

\begin{abstract}
Non-alcoholic fatty liver disease (NAFLD) is now the most common cause of liver disease worldwide, and represents an increasingly important cause of hepatocellular carcinoma (HCC). As the prevalence of NAFLD has increased, the burden of NAFLD-related HCC has been rising in parallel. This is particularly evident in Western countries, where NAFLD is estimated to account for 10\%-59\% of all HCC. NAFLD-related HCC can occur in the presence or absence of cirrhosis, and, while those with cirrhosis remain at the greatest risk, factors such as steatohepatitis, age, genetic polymorphisms, type 2 diabetes mellitus and obesity also appear have an impact on the risk of developing HCC in NAFLD. In this review, we present the epidemiology of NAFLD-related HCC from a Western perspective, highlighting gaps in current knowledge and future directions for research in this field.
\end{abstract}

Keywords: Non-alcoholic fatty liver disease, steatohepatitis, cirrhosis, hepatocellular carcinoma, epidemiology

\section{INTRODUCTION}

Over the past two decades, non-alcoholic fatty liver disease (NAFLD) has rapidly become the most common cause of liver disease worldwide, affecting approximately a quarter of the global population ${ }^{[1]}$. It is considered to be a hepatic end organ effect of the metabolic syndrome and its rise to prominence has 
coincided with the onset of the obesity and type 2 diabetes mellitus (T2DM) epidemics ${ }^{[2]}$. Previously, due to the strong association between NAFLD and the metabolic syndrome, NAFLD was considered a disease of affluent nations; however, with the epidemiological transition and increasing urbanisation in low and middle income countries, prevalence of the metabolic syndrome and NAFLD is now increasing rapidly across the globe $e^{[3-5]}$. This high prevalence and subsequent burden of progressive liver disease has resulted in NAFLD becoming an increasingly important cause of hepatocellular carcinoma $(\mathrm{HCC})^{[6-8]}$. This review outlines the epidemiology of NAFLD-related HCC, with a focus on Western countries.

\section{METHODS}

We conducted a search of the Medline, PubMed and Cochrane databases to identify English language, original research articles conducted in adults over the age of 18, and published between 1 January 1990 and 1 September 2019 using the Medical Subject Headings "Nonalcoholic Fatty Liver Disease" and "Hepatocellular carcinoma". Duplicate results were removed using Endnote X9, before screening by title and abstract was performed to find research relating to HCC in patients with NAFLD (single reviewer AF). Original research articles were eligible for selection, including meta-analyses, controlled trials, cohort studies and case-series, which clearly described their study population including their diagnostic criteria for NAFLD, and described the incidence or prevalence of NAFLD-related HCC.

\section{NAFLD, nonalcoholic steatohepatitis and the natural history of disease}

NAFLD is a broad term used to describe a spectrum of liver diseases characterised by excessive hepatic fat accumulation with associated insulin resistance, in the absence of a secondary cause or significant alcohol consumption $^{[9,10]}$. More precisely, NAFLD is defined as the presence of steatosis in $>5 \%$ of hepatocytes histologically or by proton density fat fraction on magnetic resonance imaging (MRI-PDFF), and it can be subclassified into nonalcoholic fatty liver (NAFL) or nonalcoholic steatohepatitis $(\mathrm{NASH})^{[9,10]}$. While NAFL refers to simple steatosis, NASH is characterised by hepatic lobular inflammation and is associated with an increased risk of progression to fibrosis, cirrhosis and hepatic decompensation ${ }^{[9]}$. NASH can only reliably be differentiated from NAFL histologically, and the prevalence of NASH in liver biopsies from NAFLD patients is estimated to be $59.10 \%$ (95\%CI: $47.55 \%-69.73 \%$ ) from pooled NASH prevalence data ${ }^{[1]}$. This estimate is higher than previous estimates, suggesting NASH occurred in approximately one third of NAFLD patients; however, retrospective biopsy studies are inherently affected by selection bias of patients in whom there is high clinical suspicion for steatohepatitis ${ }^{[11,2]}$.

Fibrosis is an important prognostic factor in NAFLD. Fibrosis stage is independently associated with mortality, liver transplantation and liver-related events, with fibrosis occurring at twice the rate in patients with NASH compared with NAFL ${ }^{[13,14]}$. Furthermore, liver-related mortality increases significantly with increasing fibrosis stage ${ }^{[15]}$. In a meta-analysis of 411 patients from 11 cohort studies with biopsy proven NAFLD (2145.5 person-years of follow-up), the annual fibrosis progression rate in patients with NAFL and stage 0 fibrosis at baseline was 0.07 stages per year (95\%CI: 0.02-0.11 stages/year), and 0.14 stages per year in NASH (95\%CI: 0.07-0.21 stages per year) ${ }^{[16]}$. This is the equivalent of one stage of progression over 14.3 years (95\%CI: 9.1-50 years) in NAFL vs. 7.1 years (95\%CI: 4.8-14.3 years) for NASH. In patients with NASH, approximately $20 \%$ of those with advanced (F3) fibrosis will progress to cirrhosis, and $20 \%$ of compensated NASH cirrhotics will develop hepatic decompensation over a two-year period ${ }^{[17,18]}$.

\section{Current global trends in HCC}

Liver cancer, of which HCC is the main subtype, has the sixth highest cancer incidence, and is the fourth leading cause of cancer-related death worldwide ${ }^{[19]}$. There are significant geographic variations in the incidence of HCC, reflecting the differences in aetiology of liver diseases in each region ${ }^{[20]}$. In Asian countries where the highest incidence rates of HCC are reported (20 per 100,000 in men and 6.9 in 
women), viral hepatitis, particularly hepatitis B, accounts for the majority of cases with the population attributable fraction due to hepatitis B approximately $51 \%-57 \%{ }^{[21,22]}$. Comparatively, in high income regions of the world such as Western Europe, North America and Australasia, where the predominant aetiologies are hepatitis $\mathrm{C}$ and alcohol, HCC incidence rates are much lower ${ }^{[8]}$. In Europe, the age-standardised incidence rate is estimated at 6.8 per 100,000 population in men and 2.2 in women, while, in the US, the overall incidence is 11.6 per 100,000 population in men and 4.3 per 100,000 in women ${ }^{[21,23]}$. This is on the background of an almost three-fold increase in HCC cases in the United States over the past two decades ${ }^{[24,25]}$. Australia and New Zealand have also demonstrated a significant increase in HCC with a 7.5 -fold increase between 1982 and $2014^{[26]}$. In 2015, the Australian incidence of HCC was 7.6 per 100,000 persons, again with higher rates in men (12 per 100,000) than women (3.9 per 100,000), which is expected to have increased to 8.6 cases per 100,000 persons in $2019^{[27]}$. This increase is likely due in part to an aging population, with a high prevalence of hepatitis $\mathrm{C}$; however, the rise of the obesity epidemic, and an associated increase in the prevalence of NAFLD-related HCC, may also be contributing ${ }^{[28,29]}$. Furthermore, within these Western countries, particularly in the US, NAFLD, fibrosis and HCC are more prevalent in some ethnic sub-populations. This has been seen in Hispanic, Pacific Islander and subcontinental Indian groups, whereas other sub-populations, such as African Americans, appear to have lower rates despite similar risk factors ${ }^{[30-32]}$. Whether this difference is due to disparities in socioeconomic status or diet remains to be seen; however, there may also be inherent biological differences with a higher prevalence of the PNPLA3 genetic polymorphism noted in the Hispanic population ${ }^{[33]}$.

\section{Prevalence of NAFLD-related HCC}

NAFLD has become the most rapidly increasing cause of HCC in Western countries, with the proportion of HCC attributable to NAFLD increasing significantly over the past two decades ${ }^{[34,35]}$. This shift has mirrored the rise of obesity and the metabolic syndrome, while also coinciding with the development of effective treatments for viral hepatitis and greater coverage of hepatitis B vaccination programmes ${ }^{[6]}$. This increase in NAFLD-related HCC is most pronounced in Western nations: in the BRIDGE study, a multiregional, large-scale longitudinal cohort study of consecutive newly diagnosed HCC cases, NAFLD accounted for $10 \%-12 \%$ of HCC cases in North America and Europe, but only 1\%-6\% in Asian countries ${ }^{[7]}$. In North America, NAFLD is a common cause of HCC. In a large early cohort study of HCC patients identified from a healthcare claims database in the USA, NAFLD was found to be the most common aetiology, accounting for $59 \%$ of their 4406 cases $^{[36]}$. However, subsequent similar US-based longitudinal cohort studies have reported a much lower proportion of NAFLD-related HCC. The Surveillance, Epidemiology, and End Results (SEER) cancer registry database found that NAFLD was the predominant aetiology in $14.1 \%$ of their 4929 HCC cases; this was even lower in the Veteran Affairs (VA) Hospitals cohort, where 8\% of 1500 HCCs were attributable to NAFLD ${ }^{[8,37]}$. This may reflect differences in the diversity of the groups studied here, with the VA group enriched with older males with hepatitis C. NAFLD is now the second leading cause of liver transplantation for HCC in the United States after Hepatitis C virus (HCV), and is the most rapidly growing indication for HCC-related liver transplantation, having increased from $8.3 \%$ in 2002 to $13.5 \%$ in $2012^{[38]}$. Moreover, the number of people with NAFLD-related HCC on liver transplant waiting lists in the United States has also risen from $2.1 \%$ in 2002 to $17.9 \%$ in $2017^{[38,39]}$.

European and Australasian studies have also described an increase in the prevalence of NAFLD-related HCC. In the UK, between 2000 and 2010 there was a 10-fold increase in NAFLD-related HCC, with histologically or radiologically proven NAFLD accounting for $34.8 \%$ of all HCC cases in 2010 in one cohort of 633 patients ${ }^{[34]}$. Data from the European Transplant Registry also show an increase in transplantation for NAFLD-related HCC from $0.2 \%$ in 2007 to $1.2 \%$ in $2017^{[40]}$. In Australia and New Zealand, NAFLD is the third leading cause of HCC in those who underwent transplantation, and an Australian cohort of 272 HCC patients found NAFLD to be the underlying aetiology with $14 \%$ of cases ${ }^{[41,42]}$. A summary of these cohorts is outlined in Table 1. 


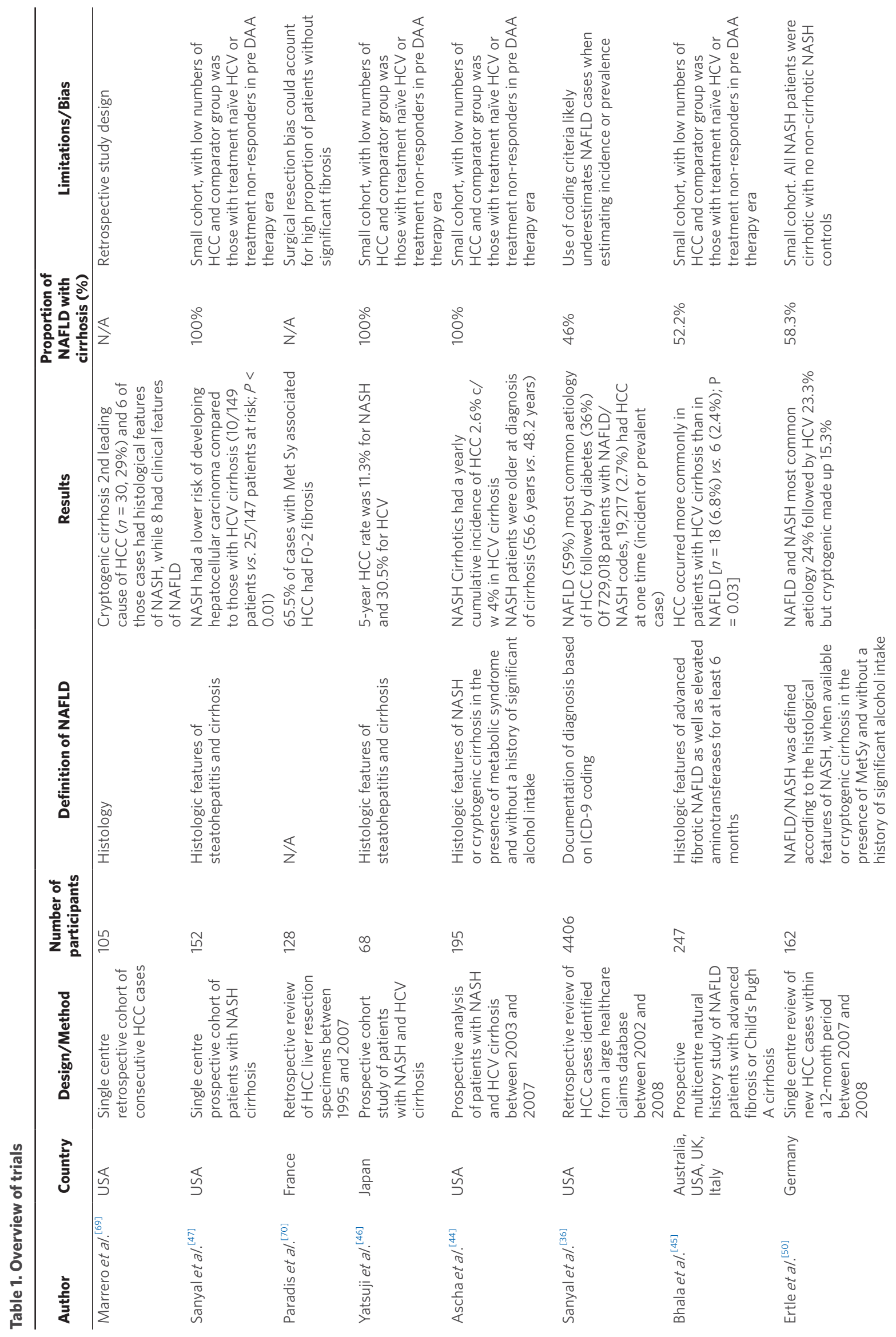



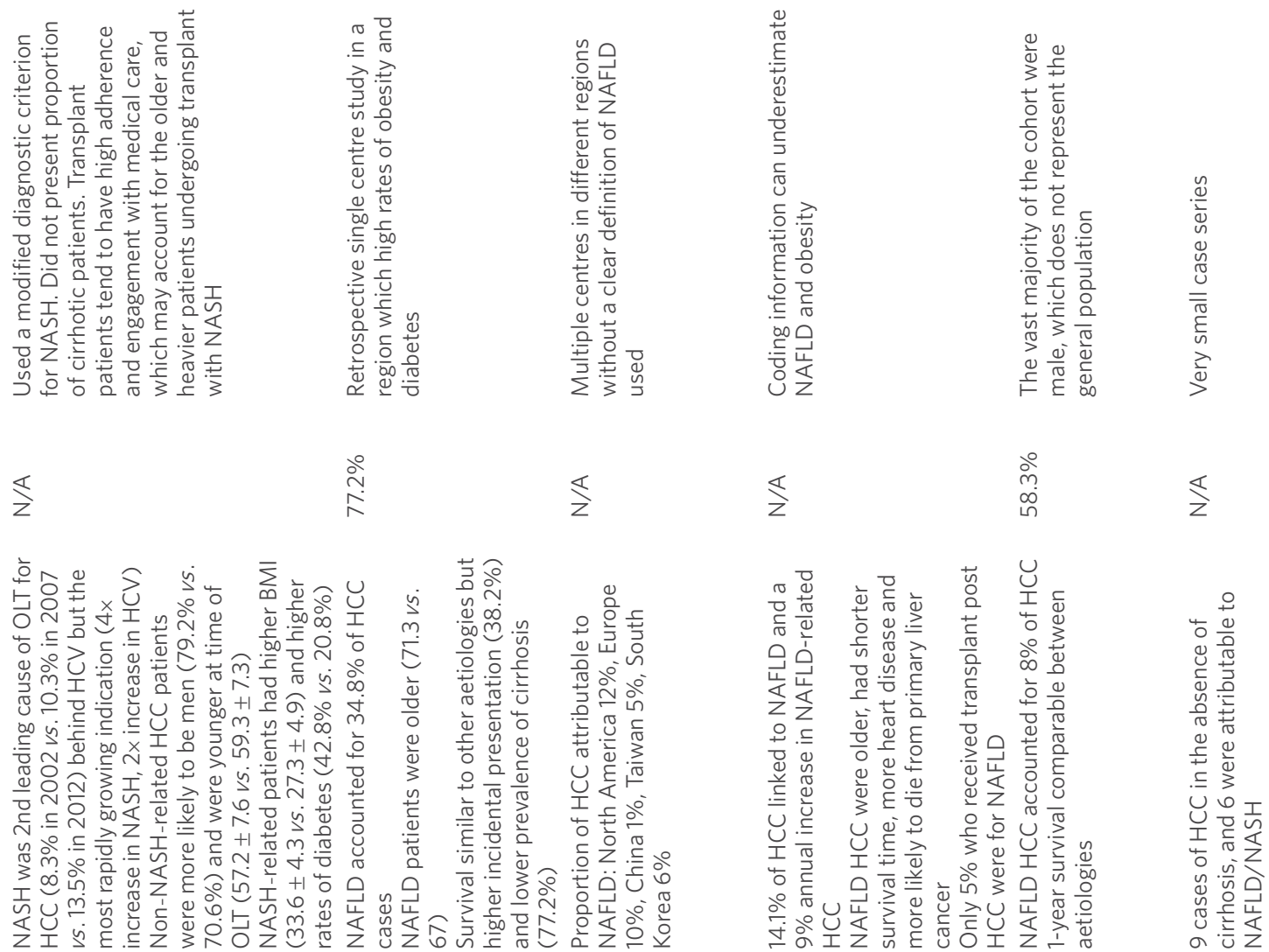

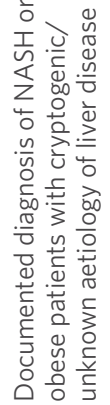
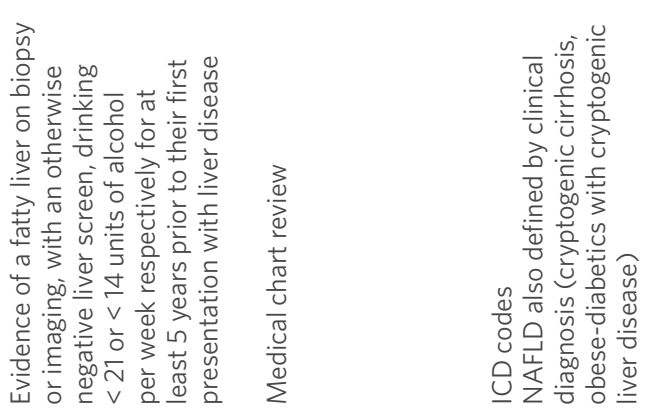

$\bar{\varnothing}$
$\stackrel{0}{0}$

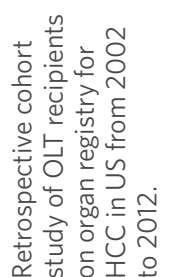

离

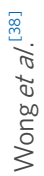

กิ

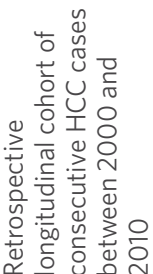

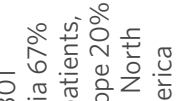

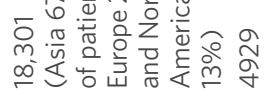

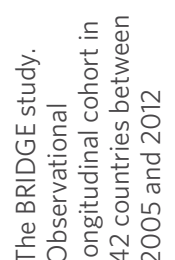

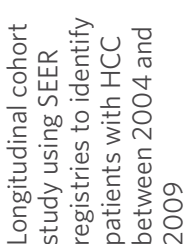

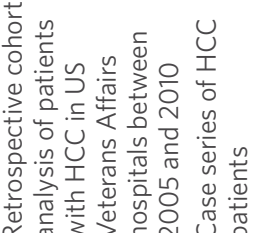

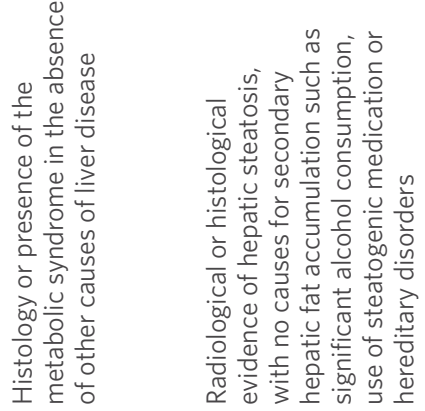

$\stackrel{\circ}{\circ}$

\&

\begin{tabular}{|c|c|c|c|c|}
\hline 6 & 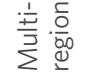 & 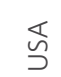 & 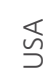 & $\underset{\nwarrow}{\longleftarrow}$ \\
\hline & 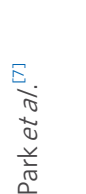 & 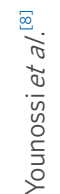 & 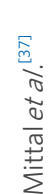 & $\overline{0}$ \\
\hline
\end{tabular}




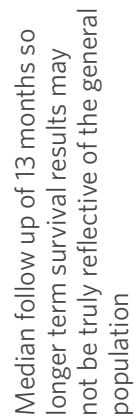

$\stackrel{0}{\infty}$
$\stackrel{n}{n ்}$

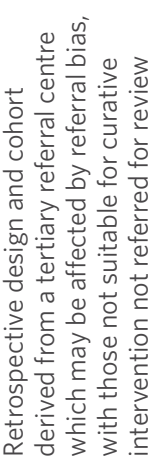

今ें

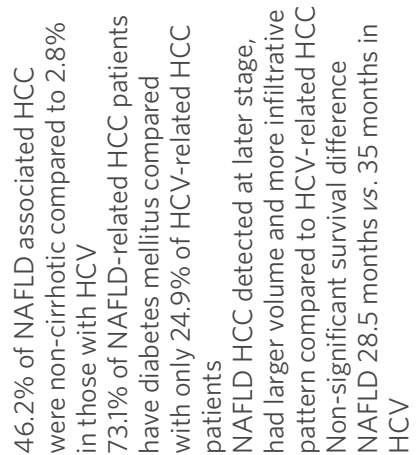

$\stackrel{\circ}{\circ}$

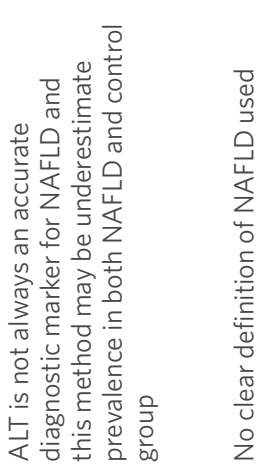

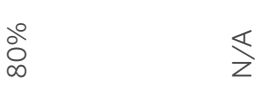
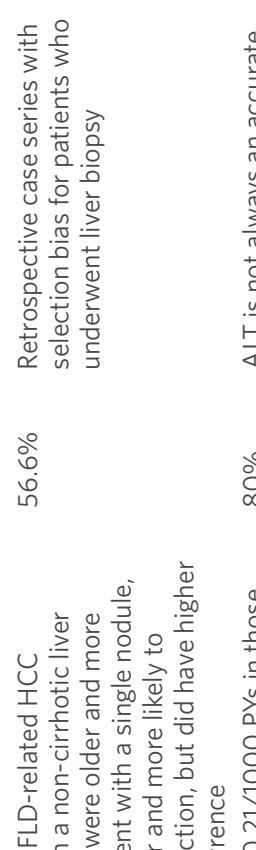

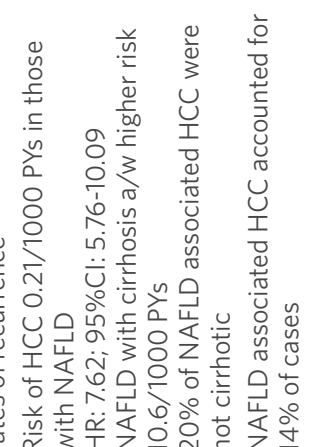

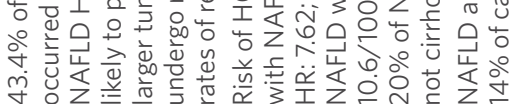

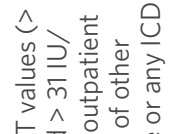

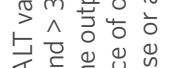

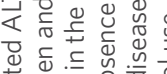

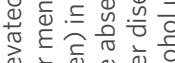

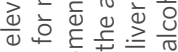

ब है ऐ.

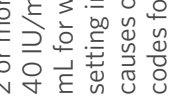
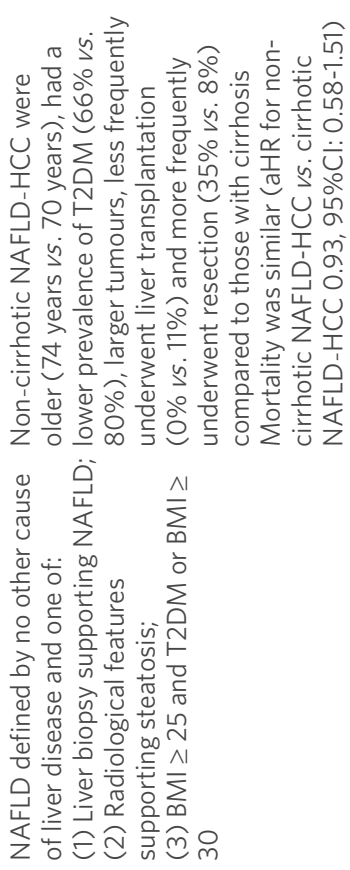

$\stackrel{\text { ป }}{\square}$

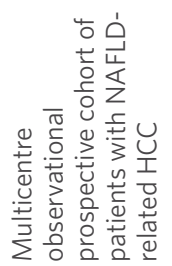

$m$

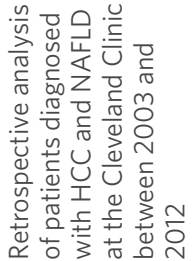

กิ
กั
ขิ

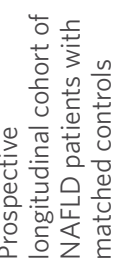

$\frac{3}{3}$
$\frac{0}{2}$
$\frac{0}{0}$
$\frac{0}{0}$
$\frac{0}{0}$
$\frac{.0}{0}$
$\frac{0}{2}$

$\stackrel{\text { N }}{\stackrel{N}{N}}$

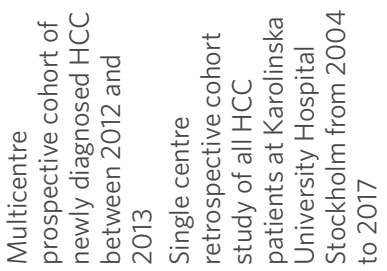

곺

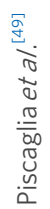

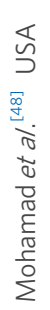

\section{这}

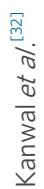

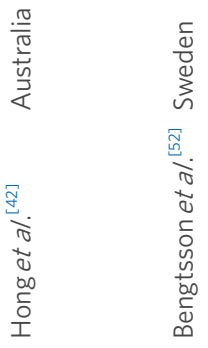

둥

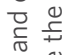

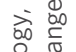

응 둔

है

음 $\frac{0}{2}$

ฮิ ํㅡㅇ

离造

जे

嵌

虫.

旁章

บ

党

范 $\frac{1}{2}$

든 은

己 ह

I.

亭 $\frac{\pi}{2}$

岂完

है is

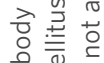

兰 $\frac{E}{\tilde{\Sigma}}$

iो

î

늠 $\sim$

这 艺

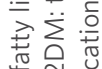

는

ํㅡㅇ

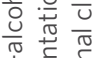

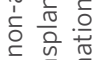

ดे 돈

这高.

$Z_{i} \geq \underline{U}$

है 능 is

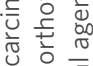

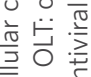

苟

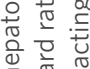

눈

ن

工舟

党莺这

崩官

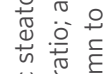

능 뭉

은

등 몽

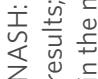




\section{Incidence of HCC in NAFLD}

Although NAFLD represents an increasing proportion of HCC cases, the true risk of HCC in NAFLD patients with and without cirrhosis is not clear. As HCC is an infrequent outcome of chronic liver disease, there are limited studies with the adequate cohort size and duration of follow up required to accurately assess incidence rates. As a result, several meta-analyses have been performed to pool data and increase the sample size. A systematic review by White $e t$ al ${ }^{[43]}$ found the risk of HCC in NAFLD patients to be in the range of $0 \%-38 \%$ over a median of 5-10 years of follow up. More recently, Younossi et al. ${ }^{[1]}$ performed a meta-analysis to evaluate the global prevalence and outcomes of NAFLD, and calculated an annual HCC incidence of 0.44 per 1000 person years (95\%CI: 0.29-0.66) in patients with NAFLD, with much higher rates in those with NASH, 5.29 per 1000 person years (95\%CI: 0.75-37.56). These meta-analyses are somewhat limited by the heterogeneity of the included studies, with small retrospective cohorts and inconsistent methods of defining NAFLD. As a result, a large United States longitudinal study led by Kanwal et al. ${ }^{[32]}$ compared 296,707 patients with NAFLD to 296,707 matched healthy controls to determine the incidence of HCC in these cohorts. NAFLD was defined by persistently elevated transaminases ( $>40 \mathrm{IU} / \mathrm{mL}$ for men and $>31 \mathrm{IU} / \mathrm{mL}$ for women, with a minimum of two results at least six months apart), in the absence of any significant alcohol intake or another cause of liver disease. The healthy controls had normal liver function tests, no other history of liver disease and minimal alcohol intake. The overall incidence of HCC in the NAFLD patients was low, 0.21 per 1000 patient years; however, HCC incidence was much higher in patients with NAFLD cirrhosis, 10.6 per 1000 patient years. Overall, cirrhosis in NAFLD appears to be the most significant risk factor for HCC [Figure 1], with approximately $6 \%-13 \%$ of patients with NAFLD cirrhosis developing a HCC during 3-10 years of follow up in a number of prospective observational cohorts ${ }^{[4-47]}$.

\section{NAFLD-related HCC in the absence of cirrhosis}

NAFLD patients without liver cirrhosis are also at increased risk of HCC compared to those without liver disease ${ }^{[32,43]}$. This is particularly concerning given the high population prevalence of NAFLD, and the implications for HCC screening and increasing disease burden ${ }^{[1]}$. In the US, Sanyal et al.$^{[36]}$ found that, among patients with NAFLD-related HCC, as many as 54\% were not known to be cirrhotic based on health care coding information. The proportion of non-cirrhotic patients was similarly high in the smaller Cleveland clinic cohort, which found that $43.4 \%$ of cases occurred in the absence of cirrhosis ${ }^{[48]}$. Those patients tended to be older $(67.5 \pm 12.3$ years $v s .62 .7 \pm 8.1$ years), and less likely to be obese $(52 \% v s .83 \%)$ or have type 2 diabetes ( $38 \%$ vs. $83 \%$ ), than their cirrhotic counterparts. Similar findings have also been reported from European cohort studies, with a high proportion of NAFLD-related HCC occurring in noncirrhotic patients: $46.2 \%$ in a multicentre Italian study, $41.7 \%$ in a single centre German study and $22.7 \%$ in a UK-based cohort ${ }^{[34,4,50]}$. In the cohort study by Kanwal et al. ${ }^{[32]}$, the risk of HCC in non-cirrhotic NAFLD was higher than in the healthy controls, but the overall incidence rates were low $(0.08 v s .0 .02$ per 1000 person years). Despite this, due to the high global prevalence of NAFLD, the absolute burden of NAFLDrelated HCC, including those with and without cirrhosis, remains significant.

\section{Clinical outcomes in NAFLD-related HCC}

Patients with NAFLD-related HCC tend to present at an older age compared to patients with HCC due to other aetiologies ${ }^{[8,28,31]}$. In the SEER cohort, NAFLD-related HCC patients were older $(73 \pm 8$ years $v s$. $66 \pm 11$ years), had more heart disease (35.1\% vs. 7\%-27\%) and had a shorter mean survival time (14.17 \pm 17.14 months vs. $17.85 \pm 21.47$ months), compared to other aetiologies; and $84.3 \%$ died of their primary liver cancer $^{[8]}$. This group found higher odds of one-year mortality (OR: 1.21, 95\%CI: 1.01-1.45) in those with NAFLD-related HCC, although other large HCC cohorts have found no difference in overall survival ${ }^{[34,37,49]}$. Furthermore, a Canadian study of 929 patients who underwent a transplant for HCC, including 60 with NAFLD-related HCC, also found that these patients had similar one-, three- and five-year survival outcomes (98\%, 96\% and 80\%, respectively) compared to non-NAFLD HCC (95\%, 84\% and 78\%, respectively), with no difference in tumour recurrence $(13.3 \% v s .14 \%)^{[51]}$. As patients with NAFLD-related 


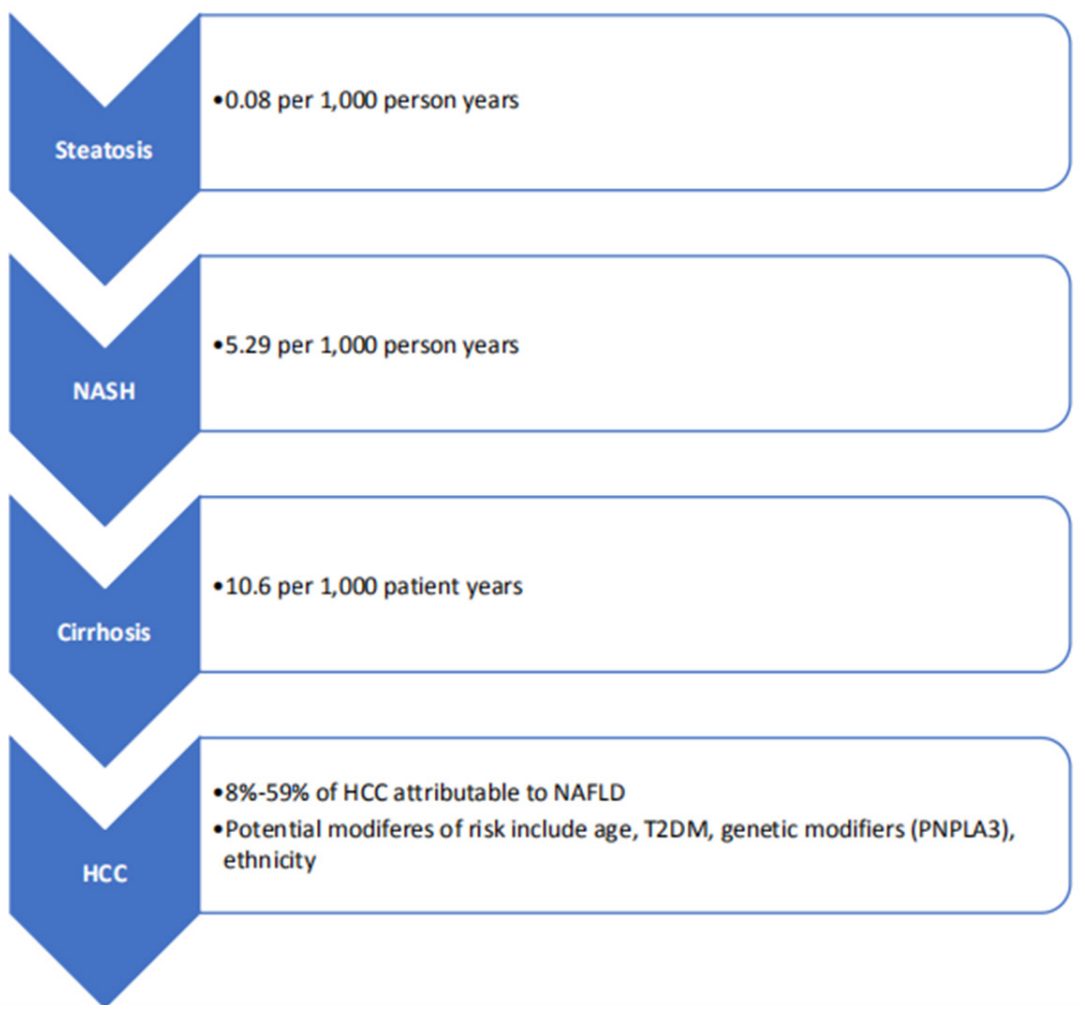

Figure 1. Increasing risk of HCC with stage of disease ${ }^{[1,7,32,36-38]}$. NASH: nonalcoholic steatohepatitis; HCC: hepatocellular carcinoma; NAFLD: non-alcoholic fatty liver disease; T2DM: type 2 diabetes mellitus

HCC tend to be older, with greater metabolic and vascular comorbidities than those presenting with HCC from other causes, the relative equivalence in survival may be due to the proportion of patients without cirrhosis in some of these cohorts $(22.8 \% \text { and } 41.7 \% \text { in the studies by Dyson and Mittal, respectively })^{[8,34,37]}$.

Although non-cirrhotic and cirrhotic NAFLD-related HCC may present differently, overall survival rates between these two groups appear to be the same. A review of NAFLD-related HCC cases at the Cleveland clinic found that HCC in non-cirrhotic patients was more likely to present as a single nodule (80.6\% vs. $52.2 \%)$ and with a larger nodule size $(>5 \mathrm{~cm})(77.8 \% v s .10 .6 \%)^{[48]}$. From a treatment perspective, they were more likely to receive hepatic resection $(66.7 \% v s .17 \%)$ but less likely to receive loco-regional therapy (22.3\% vs. 61.7\%) or orthotopic liver transplantation (OLT) ( $0 \% v s .72 .3 \%)$. They also found that HCC in non-cirrhotic NAFLD had a higher rate of recurrence than in those with cirrhosis ( $86 \% v s .14 \%)$; however, after adjusting for age or OLT, there was no difference in overall survival between the two groups. This is consistent with data from a large European cohort of NAFLD-related HCC patients in Sweden, which found non-cirrhotic patients to be older (median 74 years $v s .70$ years), with larger tumours ${ }^{[52]}$. Similarly, in this group, those without cirrhosis were more likely to have a resection ( $35 \% v s .8 \%)$, and less frequently underwent OLT ( $0 \% v s .11 \%)$, but no significant difference was found in the overall mortality rates [adjusted hazard ratio (aHR): 0.93, 95\%CI: 0.58-1.51, $P=0.78$ ]. On further analysis, parameters independently associated with a higher risk for overall mortality in non-cirrhotic patients included; Barcelona Clinic Liver Cancer class C or D (aHR compared with class 0:7.03, 95\%CI: 2.89-17.11, $P<0.001$ ), the presence of four or more tumours (aHR compared with one tumour: 9.26, 95\%CI: 3.20-26.81, $P<$ 0.001 ), higher serum albumin (aHR 0.29 per increased g/dL, 95\%CI: $0.15-0.56, P<0.001$ ) and the presence of T2DM (aHR: 3.65, 95\%CI: 1.69-7.89, $P=0.001$ ). 


\section{Obesity and type 2 diabetes and HCC risk}

Obesity and T2DM are metabolic comorbidities closely associated with NAFLD, which have also been independently linked to the development of $\mathrm{HCC}^{[53,54]}$. T2 DM has been found to be associated with increased HCC risk, particularly in patients with non-viral liver disease. A 2012 meta-analysis including 17 case-control studies and 32 cohort studies showed a statistically significant increased risk of HCC prevalence among diabetic individuals (RR: $2.31,95 \% \mathrm{CI}: 1.87-2.84)^{[53]}$. The pooled risk estimate from 17 case-control studies (OR: 2.40, 95\%CI: 1.85-3.11) was slightly higher than that from the 25 cohort studies included (RR: 2.23 , 95\%CI: 1.68-2.96). This analysis was limited by lower patient numbers, which prevented adequate subgroup analysis, and, while there was some adjustment for the presence of viral hepatitis and cirrhosis, the possible presence of underlying NAFLD was not accounted for. In a study from the Mayo Clinic, the hazard ratio of HCC in cirrhotic patients without HCV infection was found to be two times higher in patients with T2DM compared to those without [Hazard Ratio (HR): 2.1, 95\%CI: 1.1-4.1] $]^{[55]}$. Additionally, T2DM has also been found to be an independent risk factor for HCC in patients with cryptogenic cirrhosis; however, these patients also demonstrated features of NAFLD based on markers of insulin resistance and lipid profiles ${ }^{[56]}$. Overall, while T2DM does appear to be associated with an increased risk of HCC in those with or without known liver disease, current studies are limited by confounding and the difficulty of excluding underlying NAFLD as a contributing factor.

Obesity is associated with an increased risk of many types of malignancy, and several epidemiological studies suggest a modest increase in the relative risk of HCC in obese populations ${ }^{[57]}$. A 2012 meta-analysis of 26 prospective cohort studies, including 25,337 individuals, found excess body weight [body mass index $\left.(\mathrm{BMI}) \geq 25 \mathrm{~kg} / \mathrm{m}^{2}\right]$ and obesity $\left(\mathrm{BMI} \geq 30 \mathrm{~kg} / \mathrm{m}^{2}\right)$ to be associated with an increased risk of primary liver cancer [Summary Relative Risks (SRR) 1.48, 95\%CI: 1.31-1.67; and SRR 1.83, 95\%CI: 1.59-2.11, respectively $]^{[54]}$. While this evidence is somewhat limited by heterogeneity, on subgroup analysis, there was an increased risk in males, those with HCV and cirrhosis from any aetiology compared with the general population, suggesting that obesity may be an important cofactor in the development of HCC.

\section{Genetic markers in NAFLD-related HCC}

Several host genetic polymorphisms have been linked to the presence of NAFLD, risk of fibrosis progression and the development of NAFLD-related $\mathrm{HCC}^{[58]}$. These are particularly relevant as they may present novel biomarkers to help triage those at most risk of HCC and therefore in need of screening, particularly among those with NAFLD and no cirrhosis. The most studied of these is a single-nucleotide polymorphism (SNP) in PNPLA3. The association between PNPLA3 (rs738409) and hepatic steatosis was first identified in the genome-wide survey performed on the $>2000$ participants of the Dallas Heart Study who underwent Magnetic Resonance Spectroscopy of the liver for quantification of liver fat ${ }^{[59]}$. The presence of this SNP has since been found to be associated with an increased risk of advanced fibrosis in all patients with liver disease, and a 2014 meta-analysis confirmed that it was also associated with an increased risk of HCC in patients with NASH and alcohol-related cirrhosis (OR: 1.67, 95\%CI: 1.27-2.21), but not in other aetiologies (OR: 1.33, 95\%CI: 0.96-1.82) ${ }^{[60]}$. Moreover, in a large well-characterised Northern European cohort of patients with histologically proven NAFLD patients, 100 of whom had NAFLD-related HCC, it was shown that PNPLA3 rs738409, especially the C > G polymorphism, was associated with an increased risk of HCC independent of other traditional risk factors including age, gender, BMI, presence of T2DM or the presence of advanced fibrosis or cirrhosis ${ }^{[61]}$. Despite this evidence, the potential use of this SNP as a predictive biomarker to identify high-risk individuals for HCC screening has not yet been validated in larger cohorts, and it is not widely used.

Other potential genetic risk modifiers of interest include a variant in the MBOAT7 gene, which was associated with an increased risk of NAFLD-related HCC in an Italian cohort of NAFLD patients (OR: 1.65, 95\%CI: $1.08-2.55 ; n=765)$, particularly in those without advanced fibrosis $(P<0.001)$, and TERT 
promoter mutations that can be found in low and high grade dysplastic lesions, and in increased frequency in early $\mathrm{HCC}^{[62-64]}$. The TM6SF2 genetic variant on chromosome 19 has also been shown to be associated with an increased risk of NAFLD and NAFLD-related hepatic fibrosis and cirrhosis, independent of other known traditional risk factors including PNPLA3 status ${ }^{[65]}$. In one cohort of 99 biopsy-confirmed HCC patients, homozygous carriage of the TM6SF2 rs58542926 minor allele was associated with an increased risk of NAFLD-related HCC. However, this was not shown to be significant on multivariate analysis when adjusting for traditional risk factors such as age, gender or cirrhosis ${ }^{[66]}$. Further investigation is required to determine the clinical significance of these associations and establish whether they may be of prognostic or diagnostic significance in the future. Consideration of cost, access to and acceptability of genetic sequencing as a screening or prognostic tool for patients with NAFLD must also be explored.

\section{Should patients with NAFLD undergo HCC surveillance?}

Routine HCC surveillance is recommended for all patients with cirrhosis regardless of the aetiology of their liver disease ${ }^{[9,10]}$, and as such all patients with NAFLD cirrhosis should be enrolled in a screening program. While HCCs that are detected by surveillance programs are found at an earlier stage than those found incidentally, patients with NAFLD-related HCC are less likely to have recognised liver disease, and as such have a lower proportion of HCCs detected through screening compared with other causes of liver disease ${ }^{[67]}$. In the UK Newcastle cohort and the HCC NAFLD Italian groups, only $22.8 \%-47.6 \%$ of NAFLD-related HCC cases were detected during surveillance compared with $46.2 \%-63.3 \%$ in $\mathrm{HCV}^{[34,49]}$. Additionally, even in high risk patients who have NAFLD cirrhosis, screening is less likely to detect early stage HCCs compared with other aetiologies ${ }^{[34,37,49]}$. This is likely attributable to the current recommended screening modality of ultrasound, which can be technically challenging in obese patients with excess visceral adiposity, and is highly operator dependant ${ }^{[68]}$. Cross-sectional imaging such as MRI may be used as an alternative in patients in whom adequate views of the liver are unable to be obtained via ultrasound, however the suitability and cost-effectiveness of this approach has yet to be determined. Concern remains regarding whether NAFLD patients without cirrhosis should also be screened, considering that $23 \%-54 \%$ of NAFLD-related HCC occurs in non-cirrhotic patients. However, due to the large volume of NAFLD patients and low annual HCC incidence of less than $2 \%$ in this cohort, routine screening is not currently recommended $^{[34,36,37,49]}$. The development of risk stratification tools may be of use in identifying high-risk patients who would benefit from screening, and may include the use of genetic markers.

\section{Limitations in current estimates and Future directions}

While these observational studies are informative in reviewing trends, there are several limitations to this method of determining the true burden of disease. The largest cohorts have typically identified NAFLD using coded registry data, or blood tests, which can be inconsistent and will not always reflect whether there is underlying fibrosis or steatohepatitis. Additionally, HCC registry information tends to be more representative of tertiary care and patients who have been referred for active management. As NAFLDrelated HCC patients tend to be older and more comorbid, it is possible that not all cases are referred to these specialist centres, and they may have remained in community care settings. Similarly, as the SEER cohort demonstrated, patients with NAFLD-related HCC are less likely to be transplanted compared to HCC due to other causes, thus transplant registry information is also likely to underestimate the true burden of disease. Another limitation of cross-sectional retrospective studies is the evolving nature of NAFLD and NASH. These are not static disease states, but can progress or regress over time with changes in weight, which is often difficult to assess. While these studies have examined the risk associated with NAFLD alone, the increasing prevalence of obesity and diabetes in HCC of all aetiologies suggests that these metabolic factors including NAFLD could be an important co-factor in hepatic carcinogenesis ${ }^{[34]}$. In the future, the development of a simple, cost-effective population-based diagnostic test would be of benefit to improve the detection rate of NAFLD and trigger screening for underlying fibrosis and cirrhosis. Additionally, the development of prognostic markers or algorithms to identify high-risk patients for HCC surveillance is also an area of ongoing research. Further, as treatment for NAFLD progresses, and weight 
management becomes a greater focus of therapy, the ongoing risk of HCC in individuals with NAFLD who have subsequently lost weight or undergone therapy will need to be evaluated.

\section{CONCLUSION}

NAFLD is an important cause of liver disease worldwide and is associated with an increased risk of developing HCC, particularly in the presence of liver cirrhosis. While the absolute risk appears to be low, prevalence of NAFLD-related HCC is increasing in parallel with the prevalence of NAFLD worldwide, particularly in the West. The future global burden of NAFLD-related HCC represents a major public health threat and further research to identify cost-effective prevention and treatment strategies are urgently required.

\section{DECLARATIONS}

\section{Authors' contributions}

Performed the literature review and generated the manuscript: Farrell A

Assisted in developing the outline for the manuscript: Howell J

Responsible for review of the manuscript: Ryan M, Howell J

\section{Availability of data and materials}

Not applicable.

\section{Financial support and sponsorship}

None.

\section{Conflicts of interest}

All authors declared that there are no conflicts of interest.

\section{Ethical approval and consent to participate}

Not applicable.

\section{Consent for publication}

Not applicable.

\section{Copyright}

(c) The Author(s) 2020.

\section{REFERENCES}

1. Younossi ZM, Koenig AB, Abdelatif D, Fazel Y, Henry L, et al. Global epidemiology of nonalcoholic fatty liver disease-Meta-analytic assessment of prevalence, incidence, and outcomes. Hepatology 2016;64:73-84.

2. Kim D, Touros A, Kim WR. Nonalcoholic fatty liver disease and metabolic syndrome. Clin Liver Dis 2018;22:133-40.

3. Estes C, Anstee QM, Arias-Loste MT, Bantel H, Bellentani S, et al. Modeling NAFLD disease burden in China, France, Germany, Italy, Japan, Spain, United Kingdom, and United States for the period 2016-2030. J Hepatol 2018;69:896-904.

4. Ford ES, Giles WH, Mokdad AH. Increasing prevalence of the metabolic syndrome among u.s. Adults. Diabetes Care 2004;27:2444-9.

5. Saklayen MG. The global epidemic of the metabolic syndrome. Curr Hypertens Rep 2018;20:12.

6. Bertuccio P, Turati F, Carioli G, Rodriguez T, La Vecchia C, et al. Global trends and predictions in hepatocellular carcinoma mortality. J Hepatol 2017;67:302-9.

7. Park JW, Chen M, Colombo M, Roberts LR, Schwartz M, et al. Global patterns of hepatocellular carcinoma management from diagnosis to death: the BRIDGE Study. Liver Int 2015;35:2155-66.

8. Younossi ZM, Otgonsuren M, Henry L, Venkatesan C, Mishra A, et al. Association of nonalcoholic fatty liver disease (NAFLD) with hepatocellular carcinoma (HCC) in the United States from 2004 to 2009. Hepatology 2015;62:1723-30.

9. European Association for the Study of the Liver (EASL); European Association for the Study of Diabetes (EASD); European Association 
for the Study of Obesity (EASO). EASL-EASD-EASO Clinical Practice Guidelines for the management of non-alcoholic fatty liver disease. J Hepatol 2016;64:1388-402.

10. Chalasani N, Younossi Z, Lavine JE, Charlton M, Cusi K, et al. The diagnosis and management of nonalcoholic fatty liver disease: practice guidance from the American Association for the Study of Liver Diseases. Hepatology 2018;67:328-57.

11. Williams CD, Stengel J, Asike MI, Torres DM, Shaw J, et al. Prevalence of nonalcoholic fatty liver disease and nonalcoholic steatohepatitis among a largely middle-aged population utilizing ultrasound and liver biopsy: a prospective study. Gastroenterology 2011;140:124-31.

12. Farrell GC, Larter CZ. Nonalcoholic fatty liver disease: from steatosis to cirrhosis. Hepatology 2006;43:S99-112.

13. Ekstedt M, Hagström H, Nasr P, Fredrikson M, Stål P, et al. Fibrosis stage is the strongest predictor for disease-specific mortality in NAFLD after up to 33 years of follow-up. Hepatology 2015;61:1547-54.

14. Angulo P, Kleiner DE, Dam-Larsen S, Adams LA, Bjornsson ES, et al. Liver fibrosis, but no other histologic features, is associated with long-term outcomes of patients with nonalcoholic fatty liver disease. Gastroenterology 2015;149:389-97.e10.

15. Dulai PS, Singh S, Patel J, Soni M, Prokop LJ, et al. Increased risk of mortality by fibrosis stage in nonalcoholic fatty liver disease: systematic review and meta-analysis. Hepatology 2017;65:1557-65.

16. Singh S, Allen AM, Wang Z, Prokop LJ, Murad MH, et al. Fibrosis progression in nonalcoholic fatty liver vs. nonalcoholic steatohepatitis: a systematic review and meta-analysis of paired-biopsy studies. Clin Gastroenterol Hepatol 2015;13:643-54.e1-9.

17. Loomba R, Adams LA. The $20 \%$ rule of NASH progression: the natural history of advanced fibrosis and cirrhosis caused by NASH. Hepatology 2019;70:1885-88.

18. Sanyal AJ, Harrison SA, Ratziu V, Abdelmalek MF, Diehl AM, et al. The natural history of advanced fibrosis due to nonalcoholic steatohepatitis: data from the simtuzumab Trials. Hepatology 2019;70:1913-27.

19. Fitzmaurice C, Allen C, Barber RM, Barregard L, Bhutta ZA, et al.; Global Burden of Disease Collaboration. Global, regional, and national cancer incidence, mortality, years of life lost, years lived with disability, and disability-adjusted life-years for 32 cancer groups, 1990 to 2015: a systematic analysis for the global burden of disease study. JAMA Oncol 2017;3:524-48.

20. Bray F, Ferlay J, Soerjomataram I, Siegel RL, Torre LA, et al. Global cancer statistics 2018: GLOBOCAN estimates of incidence and mortality worldwide for 36 cancers in 185 countries. CA Cancer J Clin 2018;68:394-424.

21. Wong MC, Jiang JY, Goggins WB, Liang M, Fang Y, et al. International incidence and mortality trends of liver cancer: a global profile. Sci Rep 2017;7:45846.

22. Baecker A, Liu X, La Vecchia C, Zhang ZF. Worldwide incidence of hepatocellular carcinoma cases attributable to major risk factors. Eur J Cancer Prev 2018;27:205-12.

23. Kim D, Li AA, Perumpail BJ, Gadiparthi C, Kim W, et al. Changing trends in etiology-based and ethnicity-based annual mortality rates of cirrhosis and hepatocellular carcinoma in the United States. Hepatology 2019;69:1064-74.

24. El-Serag HB, Kanwal F. Epidemiology of hepatocellular carcinoma in the United States: where are we? Where do we go? Hepatology 2014;60:1767-75.

25. Altekruse SF, McGlynn KA, Reichman ME. Hepatocellular carcinoma incidence, mortality, and survival trends in the United States from 1975 to 2005. J Clin Oncol 2009;27:1485-91.

26. Wallace MC, Preen DB, Short MW, Adams LA, Jeffrey GP. Hepatocellular carcinoma in Australia 1982-2014: increasing incidence and improving survival. Liver Int 2019;39:522-30.

27. Australian Institute of Health and Welfare. Cancer in Australia 2019. Available from: https://www.aihw.gov.au/reports/cancer/cancer-inaustralia-2019/formats [Last accessed on 12 Mar 2020]

28. Hajarizadeh B, Grebely J, Dore GJ. Epidemiology and natural history of HCV infection. Nat Rev Gastroenterol Hepatol 2013;10:553-62.

29. Loomba R, Sanyal AJ. The global NAFLD epidemic. Nat Rev Gastroenterol Hepatol 2013;10:686-90.

30. Yang B, Liu JB, So SK, Han SS, Wang SS, et al. Disparities in hepatocellular carcinoma incidence by race/ethnicity and geographic area in California: implications for prevention. Cancer 2018;124:3551-59.

31. Pham C, Fong TL, Zhang J, Liu L. Striking racial/ethnic disparities in liver cancer incidence rates and temporal trends in California, 1988-2012. J Natl Cancer Inst 2018;110:1259-69.

32. Kanwal F, Kramer JR, Mapakshi S, Natarajan Y, Chayanupatkul M, et al. Risk of hepatocellular cancer in patients with non-alcoholic fatty liver disease. Gastroenterology 2018;155:1828-37.e2.

33. Danford CJ, Yao ZM, Jiang ZG. Non-alcoholic fatty liver disease: a narrative review of genetics. J Biomed Res 2018;32:389-400.

34. Dyson J, Jaques B, Chattopadyhay D, Lochan R, Graham J, et al. Hepatocellular cancer: the impact of obesity, type 2 diabetes and a multidisciplinary team. J Hepatol 2014;60:110-7.

35. Vernon G, Baranova A, Younossi ZM. Systematic review: the epidemiology and natural history of non-alcoholic fatty liver disease and non-alcoholic steatohepatitis in adults. Aliment Pharmacol Ther 2011;34:274-85.

36. Sanyal A, Poklepovic A, Moyneur E, Barghout V. Population-based risk factors and resource utilization for HCC: US perspective. Curr Med Res Opin 2010;26:2183-91.

37. Mittal S, Sada YH, El-Serag HB, Kanwal F, Duan Z, et al. Temporal trends of nonalcoholic fatty liver disease-related hepatocellular carcinoma in the veteran affairs population. Clin Gastroenterol Hepatol 2015;13:594-601.e1.

38. Wong RJ, Cheung R, Ahmed A. Nonalcoholic steatohepatitis is the most rapidly growing indication for liver transplantation in patients with hepatocellular carcinoma in the U.S. Hepatology 2014;59:2188-95.

39. Younossi Z, Tacke F, Arrese M, Chander Sharma B, Mostafa I, et al. Global perspectives on nonalcoholic fatty liver disease and nonalcoholic steatohepatitis. Hepatology 2019;69:2672-82.

40. Belli LS, Perricone G, Adam R, Cortesi PA, Strazzabosco M, et al. Impact of DAAs on liver transplantation: Major effects on the evolution of indications and results. An ELITA study based on the ELTR registry. J Hepatol 2018;69:810-17. 
41. Calzadilla-Bertot L, Jeffrey GP, Jacques B, McCaughan G, Crawford M, et al. Increasing incidence of nonalcoholic steatohepatitis as an indication for liver transplantation in Australia and New Zealand. Liver Transpl 2019;25:25-34.

42. Hong TP, Gow PJ, Fink M, Dev A, Roberts SK, et al. Surveillance improves survival of patients with hepatocellular carcinoma: a prospective population-based study. Med J Aust 2018;209:348-54.

43. White DL, Kanwal F, El-Serag HB. Association between nonalcoholic fatty liver disease and risk for hepatocellular cancer, based on systematic review. Clin Gastroenterol Hepatol 2012;10:1342-59.e2.

44. Ascha MS, Hanouneh IA, Lopez R, Tamimi TA, Feldstein AF, et al. The incidence and risk factors of hepatocellular carcinoma in patients with nonalcoholic steatohepatitis. Hepatology 2010;51:1972-8.

45. Bhala N, Angulo P, van der Poorten D, Lee E, Hui JM, et al. The natural history of nonalcoholic fatty liver disease with advanced fibrosis or cirrhosis: an international collaborative study. Hepatology 2011;54:1208-16.

46. Yatsuji S, Hashimoto E, Tobari M, Taniai M, Tokushige K, et al. Clinical features and outcomes of cirrhosis due to non-alcoholic steatohepatitis compared with cirrhosis caused by chronic hepatitis C. J Gastroenterol Hepatol 2009;24:248-54.

47. Sanyal AJ, Banas C, Sargeant C, Luketic VA, Sterling RK, et al. Similarities and differences in outcomes of cirrhosis due to nonalcoholic steatohepatitis and hepatitis C. Hepatology 2006;43:682-9.

48. Mohamad B, Shah V, Onyshchenko M, Elshamy M, Aucejo F, et al. Characterization of hepatocellular carcinoma (HCC) in non-alcoholic fatty liver disease (NAFLD) patients without cirrhosis. Hepatol Int 2016;10:632-9.

49. Piscaglia F, Svegliati-Baroni G, Barchetti A, Pecorelli A, Marinelli S, et al. Clinical patterns of hepatocellular carcinoma in nonalcoholic fatty liver disease: a multicenter prospective study. Hepatology 2016;63:827-38.

50. Ertle J, Dechêne A, Sowa JP, Penndorf V, Herzer K, et al. Non-alcoholic fatty liver disease progresses to hepatocellular carcinoma in the absence of apparent cirrhosis. Int J Cancer 2011;128:2436-43.

51. Sadler EM, Mehta N, Bhat M, Ghanekar A, Greig PD, et al. Liver transplantation for NASH-related hepatocellular carcinoma versus nonNASH etiologies of hepatocellular carcinoma. Transplantation 2018;102:640-47.

52. Bengtsson B, Stål P, Wahlin S, Björkström NK, Hagström H. Characteristics and outcome of hepatocellular carcinoma in patients with NAFLD without cirrhosis. Liver Int 2019;39:1098-108.

53. Wang P, Kang D, Cao W, Wang Y, Liu Z. Diabetes mellitus and risk of hepatocellular carcinoma: a systematic review and meta-analysis. Diabetes Metab Res Rev 2012;28:109-22.

54. Chen Y, Wang X, Wang J, Yan Z, Luo J. Excess body weight and the risk of primary liver cancer: an updated meta-analysis of prospective studies. Eur J Cancer 2012;48:2137-45.

55. Yang JD, Mohamed HA, Cvinar JL, Gores GJ, Roberts LR, et al. Diabetes Mellitus Heightens the Risk of Hepatocellular Carcinoma Except in Patients With Hepatitis C Cirrhosis. Am J Gastroenterol 2016;111:1573-80.

56. Bugianesi E, Leone N, Vanni E, Marchesini G, Brunello F, et al. Expanding the natural history of nonalcoholic steatohepatitis: from cryptogenic cirrhosis to hepatocellular carcinoma. Gastroenterology 2002;123:134-40.

57. Pischon T, Nimptsch K. Obesity and risk of cancer: an introductory overview. Recent Results Cancer Res 2016;208:1-15.

58. Anstee QM, Reeves HL, Kotsiliti E, Govaere O, Heikenwalder M. From NASH to HCC: current concepts and future challenges. Nat Rev Gastroenterol Hepatol 2019;16:411-28.

59. Romeo S, Kozlitina J, Xing C, Pertsemlidis A, Cox D, et al. Genetic variation in PNPLA3 confers susceptibility to nonalcoholic fatty liver disease. Nat Genet 2008;40:1461-5.

60. Singal AG, Manjunath H, Yopp AC, Beg MS, Marrero JA, et al. The effect of PNPLA3 on fibrosis progression and development of hepatocellular carcinoma: a meta-analysis. Am J Gastroenterol 2014;109:325-34.

61. Liu YL, Patman GL, Leathart JB, Piguet AC, Burt AD, et al. Carriage of the PNPLA3 rs $738409 \mathrm{C}>\mathrm{G}$ polymorphism confers an increased risk of non-alcoholic fatty liver disease associated hepatocellular carcinoma. J Hepatol 2014;61:75-81.

62. Donati B, Dongiovanni P, Romeo S, Meroni M, McCain M, et al. MBOAT7 rs641738 variant and hepatocellular carcinoma in noncirrhotic individuals. Sci Rep 2017;7:4492.

63. Donati B, Pietrelli A, Pingitore P, Dongiovanni P, Caddeo A, et al. Telomerase reverse transcriptase germline mutations and hepatocellular carcinoma in patients with nonalcoholic fatty liver disease. Cancer Med 2017;6:1930-40.

64. Zucman-Rossi J, Villanueva A, Nault JC, Llovet JM. Genetic Landscape and Biomarkers of Hepatocellular Carcinoma. Gastroenterology 2015;149:1226-39.e4.

65. Zhang X, Liu S, Dong Q, Xin Y, Xuan S. The genetics of clinical liver diseases: insight into the TM6SF2 E167K variant. J Clin Transl Hepatol 2018;6:326-31.

66. Liu YL, Reeves HL, Burt AD, Tiniakos D, McPherson S, et al. TM6SF2 rs58542926 influences hepatic fibrosis progression in patients with non-alcoholic fatty liver disease. Nat Commun 2014;5:4309.

67. Taylor EJ, Jones RL, Guthrie JA, Rowe IA. Modeling the benefits and harms of surveillance for hepatocellular carcinoma: information to support informed choices. Hepatology 2017;66:1546-55.

68. Cengiz M, Sentürk S, Cetin B, Bayrak AH, Bilek SU. Sonographic assessment of fatty liver: intraobserver and interobserver variability. Int J Clin Exp Med 2014;7:5453-60.

69. Marrero JA, Fontana RJ, Su GL, Conjeevaram HS, Emick DM, et al. NAFLD may be a common underlying liver disease in patients with hepatocellular carcinoma in the United States. Hepatology 2002;36:1349-54.

70. Paradis V, Zalinski S, Chelbi E, Guedj N, Degos F, et al. Hepatocellular carcinomas in patients with metabolic syndrome often develop without significant liver fibrosis: a pathological analysis. Hepatology 2009;49:851-9.

71. Perumpail RB, Wong RJ, Ahmed A, Harrison SA. Hepatocellular carcinoma in the setting of non-cirrhotic nonalcoholic fatty liver disease and the metabolic syndrome: US experience. Dig Dis Sci 2015;60:3142-8. 